\title{
Linear and non-linear interactions between static and dynamic bifurcations of damped planar beams
}

\author{
Angelo Di Egidio ${ }^{\mathrm{a}}$, Angelo Luongo ${ }^{\mathrm{a}, *}$, Achille Paolone $^{\mathrm{b}}$ \\ a DISAT, University of L'Aquila, Monteluco di Roio, 67040 L'Aquila, Italy \\ ${ }^{\mathrm{b}}$ DISG, University of Rome "La Sapienza", via Eudossiana, 18, 00184 Rome, Italy
}

\begin{abstract}
The critical and post-critical behavior of a non-conservative non-linear structure, undergoing statical and dynamical bifurcations, is analyzed. The system consists of a purely flexible planar beam, equipped with a lumped visco-elastic device, loaded by a follower force. A unique integro-differential equation of motion in the transversal displacement, with relevant boundary conditions, is derived. Then, the linear stability diagram of the trivial rectilinear configuration is built-up in the parameter space. Particular emphasis is given to the role of the damping on the critical scenario. The occurrence of different mechanisms of instability is highlighted, namely, of divergence, Hopf, double zero, resonant and non-resonant double Hopf, and divergence-Hopf bifurcation. Attention is then focused on the two latter (codimension-two) bifurcations. A multiple scale analysis is carried-out directly on the continuous model, and the relevant non-linear bifurcation equations in the amplitudes of the two interactive modes are derived. The fixed-points of these equations are numerically evaluated as functions of two bifurcation parameters and some equilibrium paths illustrated. Finally, the bifurcation diagrams, illustrating the system behavior around the critical points of the parameter space, are obtained.
\end{abstract}

Keywords: Stability analysis; Damping effects on stability; Non-conservative loads; Bifurcation; Multiple scales method; Direct perturbation approach; Divergence and Hopf bifurcations; Beam continuous model

\section{Introduction}

Columns subjected to follower forces, after the pioneering paper by Beck [1], have recently attracted the attention of many researchers, particularly in aerospace, where tangential forces are produced by jets and rocket motors [2]. Columns with a tip mass have also been studied, to account for the influence of the mass of the device delivering the load [3]. Lumped damping forces and couples have then been considered to model the external action of air on the mass [4]. On the other hand, passive control systems for vibration reduction also demand the introduction of dissipation devices (dashpots), which can remarkably affect the critical and postcritical column behavior. The effect of dashpots on the linear stability of the Beck column has, for example, been studied in [5]; a general treatment of

\footnotetext{
* Corresponding author. Tel.: +39862 434521; fax: +39862434548.

E-mail address: luongo@ing.univaq.it (A. Luongo).
}

the effect of an added small damping on the stability of linear continuous non-conservative systems can be found in [6]. However, except for [3], where the multiple scale method has been employed to investigate supercritical and subcritical Hopf bifurcations, the attention of the aforementioned researchers has been limited to the linear stability problem. Here, it is believed important to extend the analysis to the non-linear range, accounting for possible interactions between static and dynamic bifurcation mechanism. Such a problem has been deeply studied in $[7,8]$, where some discrete versions of the continuous column have been considered.

It is well-known that the essential dynamics of a non-linear system, finite- or infinite-dimensional, close to a bifurcation point, is captured by an equivalent, lower dimensional, reduced system [9-11]. Reduction method, as linear and non-linear Galerkin, center manifold and approximate inertial manifold methods, have been thoroughly discussed in [12]. In the last decade, as an alternative and more engineering oriented 
approach, the authors have systematically applied the multiple scale method to analyze a number of bifurcations of linear codimension-one, two and three, to general finite dimensional systems [13-17]; the review paper [18] resumes their main results. More recently, they have extended the method to infinite dimensional system, to analyze divergence, Hopf and doublezero bifurcations [19-22]. The method is based on the direct treatment of the original (integro)-differential equations, avoiding any a priori discretization (as that, e.g., performed in [3]), according to the so-called direct method, widely applied by Nayfeh and co-workers [23-25], and many other authors (see, e.g., [26,27]), to several problems of non-linear dynamics.

In this paper the algorithm is applied to study a cantilever beam, constrained by a spring and two dashpot, loaded by a follower force. This system, for its simplicity, was already studied in $[19,20]$ as an example of structure undergoing divergence, Hopf and double-zero bifurcation. A deeper parametric analysis performed on the system permitted to reveal a richer bifurcation scenario, including Hopf-divergence $(\mathscr{H} \mathscr{D})$ and resonant and non-resonant double-Hopf, not discovered in the previous analysis. Therefore, the beam viscous-elastically restrained could be taken as paradigmatic system undergoing all the low-codimension bifurcations of mechanical interest. Here, the analysis of the codimension-two bifurcations is completed, while the codimension-three one (resonant Hopf-Hopf $(\mathscr{H} \mathscr{H}))$ is left for future investigation.

The paper is thus organized. In Section 2 the equations of motions are given, and detailed in Appendix A, where they are derived through a procedure alternative to that of Ref. [19]. Moreover the linear adjoint problem is defined. In Section 3 the critical scenario is depicted. Considerably emphasis is given to the influence of the relative magnitude of the two dashpots on bifurcations. The well-known destabilizing effect of damping is detected, and a new paradoxical result discovered. In Section 4 the post-critical analysis is carried-out. The bifurcation equations are first derived and then numerically studied to built-up equilibrium paths and bifurcation diagrams. Finally, in Section 5 some conclusions are drawn.

\section{Model}

A planar beam is considered, fixed at end $A$ and constrained by a linear visco-elastic device at end $B$, loaded at the tip by a follower force of intensity $P$ (Fig. 1). The device consists of an extensional spring of stiffness $k_{\mathrm{e}}$ and two dashpots of constants $c_{\mathrm{e}}$ and $c_{\mathrm{t}}$, of extensional and torsional type, respectively. The beam is assumed to be inextensible and shear-undeformable, so that bending is the unique strain measure.

\subsection{The equations of motion}

The equations of motion of the beam were derived in [19] through a variational approach by eliminating the rotation through the no-shear constraint and by using a lagrangian multiplier to account for the axial constraint. Then, after condensation of the longitudinal displacement and of the lagrangian multiplier, the following, unique, non-linear integro-differential
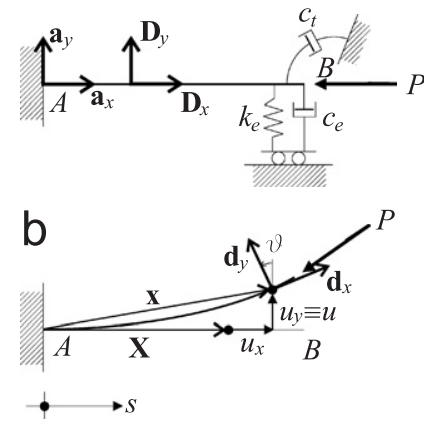

Fig. 1. Model.

equation, corrected up-to the third order, was obtained in nondimensional form:

$$
\begin{aligned}
\ddot{u}+ & u^{\prime \prime \prime \prime}+2 \mu u^{\prime \prime}+u^{\prime \prime 3}+4 u^{\prime} u^{\prime \prime} u^{\prime \prime \prime}+u^{\prime 2} u^{\prime \prime \prime \prime}+\mu\left(3 u^{\prime 2}-u_{B}^{\prime 2}\right) u^{\prime \prime} \\
& +\left[\int_{1}^{s}\left(\int_{0}^{s} \frac{1}{2} u^{\prime 2} \mathrm{~d} s\right)^{\cdot \cdot} \mathrm{d} s\right] u^{\prime \prime}+\left[\left(\int_{0}^{s} \frac{1}{2} u^{\prime 2} \mathrm{~d} s\right)^{\cdot \cdot}\right] u^{\prime}=0
\end{aligned}
$$

with the relevant boundary conditions:

$-u_{B}^{\prime \prime \prime}+\kappa u_{B}+\xi_{\mathrm{e}} \dot{u}_{B}=-\mu u_{B}^{\prime 3}+u_{B}^{\prime \prime \prime} u_{B}^{\prime 2}+u_{B}^{\prime \prime 2} u_{B}^{\prime}$,

$u_{B}^{\prime \prime}+\xi_{\mathrm{t}} \dot{u}_{B}^{\prime}=-\frac{1}{2} \xi_{\mathrm{t}} u_{B}^{\prime 2} \dot{u}_{B}^{\prime}-\frac{1}{2} u_{B}^{\prime \prime} u_{B}^{\prime 2}$,

$u_{A}=0, \quad u_{A}^{\prime}=0$,

where $A$ and $B$ denote evaluation at the beam ends. In Eqs. (1)-(2) the following non-dimensional variables and parameters were introduced:

$\tilde{t}=\omega t, \quad \tilde{s}=s / l, \quad \tilde{u}=u / l, \quad \mu=P l^{2} / 2 E I$,

$\kappa=k_{\mathrm{e}} l^{3} / E I, \quad \xi_{\mathrm{e}}=c_{\mathrm{e}} \omega l^{3} / E I, \quad \xi_{\mathrm{t}}=c_{\mathrm{t}} \omega l / E I$,

where $u(s, t) \equiv u_{y}(s, t)$ is the transversal displacement of the beam at the abscissa $s$ and time $t$ (with tilde dropped), $l$ is the length, $\omega=\left(E I / m l^{4}\right)^{1 / 2}$ is a frequency and $E I$ the bending stiffness. Moreover, $\mu$ is the load parameter, $\kappa$ is a non-dimensional stiffness parameter and $\xi_{\mathrm{e}}$ and $\xi_{\mathrm{t}}$ two non-dimensional damping coefficients, referred ahead as the 'spring stiffness' and the 'extensional and torsional damping', respectively.

To make the paper self-contained, the equations of motion (1) and (2) are reobtained in Appendix A by following a direct method, based on equilibrium and later condensation of all the internal reactive forces (axial and shear), which are not achievable by a variational procedure. The direct method, moreover, reveals the existence of an extra-term, lost in the previous formulation (namely the non-linearity affected by $\mu$ ). The slight difference in the two derivations was due to the (usually accepted) truncation at the second-order, instead of the thirdorder, of the axial strain-displacement relation used in the variational principle. A recomputation of the variational procedure, accounting for this higher-order term, permitted to recover the results of the direct method. 


\subsection{The linear direct and adjoint problems}

The linearized problem Eqs. (1)-(2) is first studied. By letting $u(s)=\phi(s) \exp (\lambda t)$, the following eigenvalue problem is obtained:

$$
\begin{aligned}
& \phi^{\prime \prime \prime \prime}+2 \mu \phi^{\prime \prime}+\lambda^{2} \phi=0, \quad \phi_{A}=0, \quad \phi_{A}^{\prime}=0, \\
& -\phi_{B}^{\prime \prime \prime}+\kappa \phi_{B}+\lambda \xi_{\mathrm{e}} \phi_{B}=0, \quad \phi_{B}^{\prime \prime}+\lambda \xi_{\mathrm{t}} \phi_{B}^{\prime}=0 .
\end{aligned}
$$

In Eq. (4), $\lambda \in \mathbb{C}$ is the eigenvalue and $\phi(s) \in \mathbb{C}$ is the associated (right) eigenvector.

It is useful to introduce for later convenience also the adjoint eigenvalue problem. It is derived from Eq. (4) performing scalar multiplication (in the complex field) by the dual variables $\psi(s) \in \mathbb{C}$ and, successively, integrating by parts (i.e. enforcing the so-called bilinear-identity). The following equations and boundary conditions was obtained:

$\psi^{\prime \prime \prime \prime \prime}+2 \mu \psi^{\prime \prime}+\bar{\lambda}^{2} \psi=0, \quad \psi_{A}=0, \quad \psi_{A}^{\prime}=0$,

$-\psi_{B}^{\prime \prime \prime}+\kappa \psi_{B}-2 \mu \psi_{B}^{\prime}+\bar{\lambda} \xi_{\mathrm{e}} \phi_{B}=0$

$\psi_{B}^{\prime \prime}+2 \mu \psi_{B}+\bar{\lambda} \xi_{\mathrm{t}} \psi_{B}^{\prime}=0$,

where the overbar denotes complex conjugate. In Eqs. (5), $\psi(s)$ is the left eigenvector associated with the eigenvalue $\lambda$ (the latter being identical in the two problems).

It should be noted that the two field equations $\left(4_{1}\right)$ and $\left(5_{1}\right)$ are identical, while the mechanical boundary conditions $\left(4_{4,5}\right)$ and $\left(5_{4,5}\right)$ differ each other; this is a consequence of the fact that the non-conservative sources (follower force and dashpots) are located at the end of the beam.

\section{Linear stability analysis}

Stability of the trivial, rectilinear configuration, of the beam is governed by the eigenvalue problem Eq. (4). It admits the eigenvector

$\phi(s)=c_{1}(\cos p s-\cosh q s)+c_{2}\left(\sin p s-\frac{p}{q} \sinh q s\right)$,

where

$q^{2}:=\sqrt{\mu^{2}-\lambda^{2}}-\mu, \quad p^{2}:=\sqrt{\mu^{2}-\lambda^{2}}+\mu$

have been set. The arbitrary constants $\mathbf{c}=\left(c_{1}, c_{2}\right)^{\mathrm{T}}$, together with the eigenvalue $\lambda$, satisfy the algebraic equations

$\mathbf{S}(\lambda) \mathbf{c}=\mathbf{0}$,

where

$\mathbf{S}(\lambda)=\left[\begin{array}{cc}-\left(\kappa+\lambda \xi_{\mathrm{e}}\right)(\cos p-\cosh q) & -\left(\kappa+\lambda \xi_{\mathrm{e}}\right)(\sin p-p / q \sinh q) \\ +p^{3} \sin p-q^{3} \sinh q, & -p^{3} \cos p-q^{2} p \cosh q \\ -\lambda \xi_{\mathrm{t}}(p \sin p+q \sinh q) & \lambda \xi_{\mathrm{t}} p(\cos p-\cosh q) \\ -p^{2} \cos p-q^{2} \cosh q, & -p^{2} \sin p-p q \sinh q\end{array}\right]$

is the 'dynamical stiffness matrix' of the system, depending on the eigenvalue $\lambda$, in addition to the system parameter, $\mu, \kappa, \xi_{\mathrm{e}}$ and $\xi_{\mathrm{t}}$.
In order to find divergence and Hopf boundaries in the parameters space, the loci of the roots $\lambda=0$ and $\lambda=\mathrm{i} \omega$ of the characteristic equation $\operatorname{det} \mathbf{S}(\lambda)=0$ must be found, respectively. In this analysis, the load $\mu$ and the stiffness $\kappa$ are taken as bifurcation parameters, while the damping coefficients $\xi_{\mathrm{e}}$ and $\xi_{\mathrm{t}}$ are assumed to be auxiliary parameters. In other words, sections $\xi_{\mathrm{e}}=$ const, $\xi_{\mathrm{t}}=$ const, of the four-dimensional parameter space are considered.

Divergence occurs at the manifold $\mathscr{D}$ on which $\operatorname{det} \mathbf{S}(0)=$ 0 . Since this equation presents an undetermined form, a limit process for $\lambda \rightarrow 0$ has to be performed. By letting $p \rightarrow \sqrt{2 \mu}$, $q \rightarrow \mathrm{i} \lambda \sqrt{2 \mu}$ in $\operatorname{det} \mathbf{S}(\lambda)$, expanding for small $\lambda$ and retaining only the leading terms, the following equation is obtained:

$2.82 \mu^{3 / 2} \kappa \cos (\sqrt{2 \mu})-5.66 \mu^{5 / 2}-2 \mu \kappa \sin (\sqrt{2 \mu})=0$

which, implicitly, defines a multi-branch curve $\mathscr{D}$ in the $(\kappa, \mu)$ plane.

Hopf bifurcation occurs at the manifold $\mathscr{H}$ on which $\operatorname{det} \mathbf{S}(\mathrm{i} \omega)=0$; in addition, $\mathfrak{R}[\partial \lambda / \partial n] \neq 0$ must hold, $\mathbf{n}$ being the normal to the curve in the plane (transversality condition). This last condition permits to exclude degenerate Hopf cases, as that ones studied by Kounadis [28]. When $\lambda=\mathrm{i} \omega$, $p$ and $q$ are real, so that the unique imaginary terms in $\mathbf{S}$ are those due to damping. By separating real and imaginary terms in the characteristic equation, two real equations of the type $f_{i}\left(\kappa, \mu, \omega ; \xi_{\mathrm{e}}, \xi_{\mathrm{t}}\right)=0(i=1,2)$ are found. They, again, implicitly define a multi-branch curve $\mathscr{H}$ in $(\kappa, \mu)$-plane, parameterized in the $\omega$-parameter. Transversality is either checked numerically, by evaluating $\mathfrak{R}[\lambda]$ closely to $\mathscr{H}$, or analytically, via an eigenvalue sensitivity analysis (see Appendix B). It is worth noting that, in general, Hopf bifurcations depend on both $\xi_{\mathrm{e}}$ and $\xi_{\mathrm{t}}$ parameters. However, when only one of the damping coefficients is different from zero, the complex characteristic equation splits in two real equations of the type $f(\kappa, \mu, \omega)=0, \xi g(\kappa, \mu, \omega)=0$ (with $\xi=\xi_{\mathrm{e}}$ or $\xi=\xi_{\mathrm{t}}$ ), i.e. the Hopf curves are independent of the (unique) damping.

To better illustrate the system behavior, and emphasize its dependence on damping, some sub-systems, obtained by removing one of both dashpots from the complete system, are preliminarily studied. All these systems, of course, exhibit the same divergence curve, while the Hopf curves change.

Fig. 2 shows the linear stability diagram of the undamped ( $\xi_{\mathrm{e}}=\xi_{\mathrm{t}}=0$ ) system, where only the (more significant) lower branches of the $\mathscr{D}$ - and $\mathscr{H}$-curves have been plotted. The Hopf curve intersects the $\mu$-axis at the well-known $\mu=10.025$ value of the Beck beam. Then, for increasing $\kappa$, the dynamical critical load increases, and the curve tangentially merges at a (degenerate) double zero $D Z$-point with the upper part of curve $\mathscr{D}$. The disappearance, for large $\kappa$ 's, of the dynamic instability is due to the fact that the system tends to the clumped-support beam, for which the load is of conservative type (see [29]).

The previous scenario abruptly changes, if an extensional damper is added (Fig. 3). Irrespective of the magnitude of $\xi_{\mathrm{e}}$ (for the reason above mentioned), the Hopf curve goes down for all values of $\kappa$, and tends to tangentially merge with the lower part of curve $\mathscr{D}$, similarly to known results $[5,30]$. Therefore, 


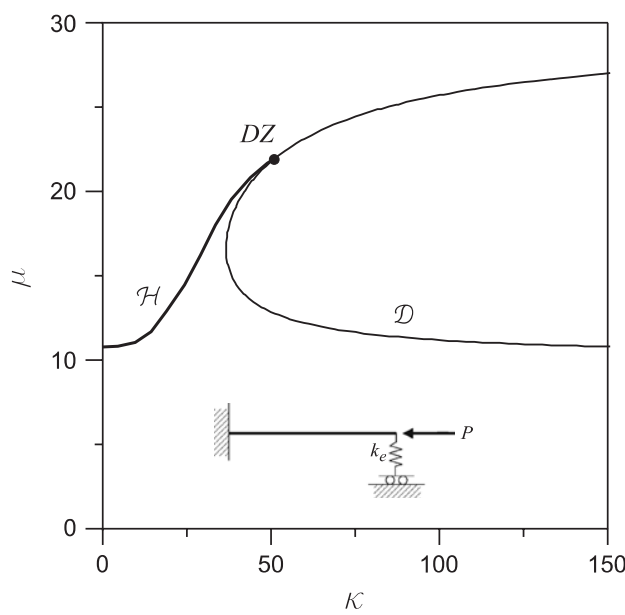

Fig. 2. Linear stability diagram for the undamped system.

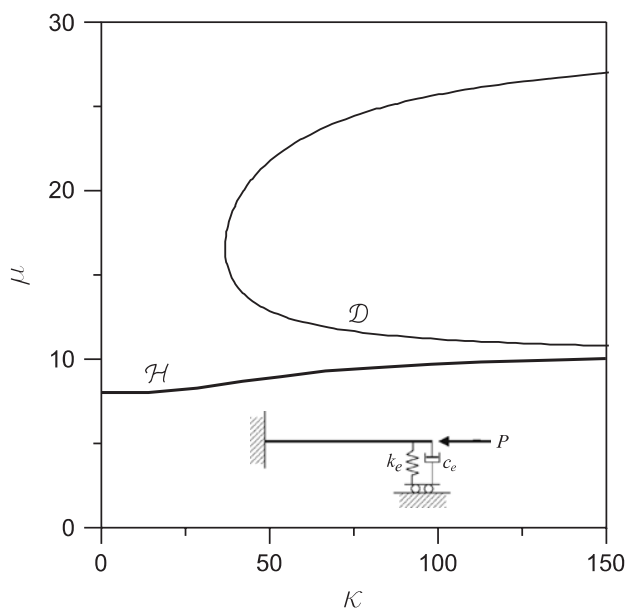

Fig. 3. Linear stability diagram for the extensionally damped system.

due to extensional damping, dynamic instability occurs also for large $\kappa$ 's. However, for small $\kappa$ 's, $\xi_{\mathrm{e}}$ has a destabilizing effect, according to the well-known Ziegler paradox.

The effect of the torsional damping, when it acts alone $\left(\xi_{\mathrm{e}}=0\right)$, is then studied (Fig. 4). First, the occurrence of Hopf bifurcations from zero values of the load $\mu$, as suggested by the numerical analysis, were analytically checked. In this special $\mu=0$ case, $p=q=\sqrt{\omega}$ (Eq. (7)), follows. The characteristic equation det $\mathbf{S}(\mathrm{i} \omega)=0$ then reduces to the very simple form $\sin ^{2} p=0$, which admits the double roots $p_{n}=n \pi(n=1,2, \ldots)$. Therefore, at special values $\kappa_{n}(n=1,2, \ldots)$ of $\kappa$, and $\mu=0$, two Hopf-curves cross each-other, with the same values of the frequency, namely $\omega_{n}=n^{2} \pi^{2}$. Thus, a infinite sequence of $1: 1$ resonant double Hopf bifurcations $(\mathscr{R} \mathscr{H})$ takes place on the $\kappa$-axis. It is worth noting that, for such sub-system, dynamic instability manifests itself both for $\mu>0$ (compressive) and $\mu<0$ (tensile) loads. The Hopf curves depicted in Fig. 4 were numerically evaluated, and turned out to be independent of $\xi_{\mathrm{t}}$. Since they cross the divergence curve and, moreover, cross each other, they reveal the existence of non-resonant $\mathscr{H} \mathscr{H}$ points and $\mathscr{H} \mathscr{D}$ points, marked in the figure. In addition, a critical point $\mathscr{H} \mathscr{H} \mathscr{D}$ of higher codimension appears, in which

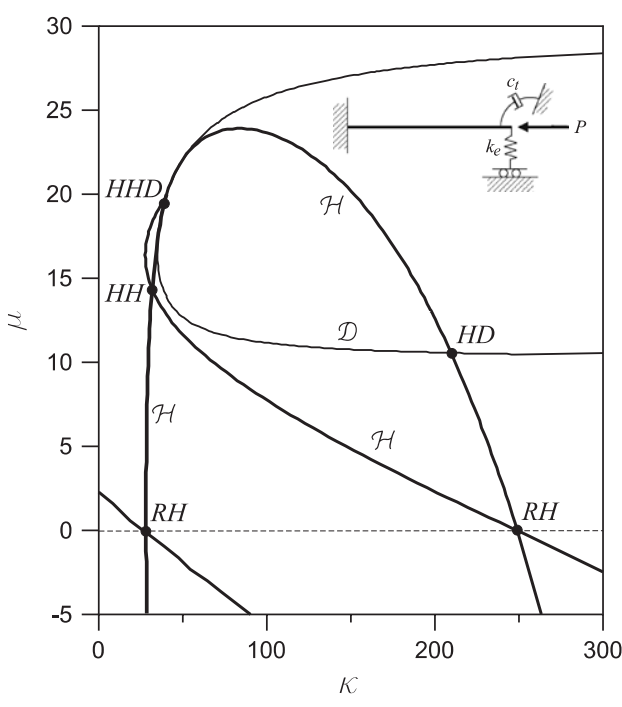

Fig. 4. Linear stability diagram for the torsionally damped system.

two Hopf curves and a divergence curve cross each other, two of them being tangent.

An other interesting aspect offered by the dynamic bifurcations of the purely torsionally damped system is the following. At a Hopf point, since the first of the two Eqs. (8) (i.e. the 'shear' boundary condition) has real coefficients (remember that $p$ and $q$ are real and $\xi_{\mathrm{e}}=0$ ), then $c_{1}$ and $c_{2}$ are real, and the eigenvector (7) is (quite unusually) also real. This entails that, in the second of Eqs. (8) (i.e. in the 'moment' boundary condition), the bending moment (real) and the damping couple (imaginary) must vanish separately. Therefore, at bifurcation, the tip of the beam does not rotate and the follower force, paradoxically behaves as a conservative force. However, this exceptional circumstance does not hold any more close to the curve $\mathscr{H}$, since perturbations of the parameters activate the nonconservative mechanism. Indeed, the transversality conditions turned out to be satisfied.

As a further step, a small extensional damping was added to the purely torsionally damped system. First, it was analytically checked that, due to $\xi_{\mathrm{e}}$, the $\mathscr{R} \mathscr{H}$ points vanish, i.e. $\xi_{\mathrm{e}}$ is an imperfection parameter for such bifurcation. A numerical analysis led to the plot of Fig. 5. This appears to be a slight perturbation of the plot of Fig. 4, if only the $\mu>0$ half-plane is considered. Hopf points was instead not found in the $\mu<0$ half-plane. The perturbation, moreover, makes the $\mathscr{H} \mathscr{H} \mathscr{D}$ point to vanish.

Comparable values of the two damping coefficients was finally considered in Figs. 6 and 7. They show (Figs. 6a and 7a) that large extensional damping does not qualitatively modify the scenario of Fig. 5 (small $\xi_{\mathrm{e}}$ ). Therefore, it can be concluded, that the system behavior is more strongly affected by torsional damping; extensional damping acts as an imperfection, destroying some bifurcation points, but slightly modifying the curves.

Figs. 6 and 7 are now analyzed in more detail. In Fig. 6a, both damping coefficients was varied, by keeping constant their ratio $\Xi:=\xi_{\mathrm{e}} / \xi_{\mathrm{t}}$. It was observed, that all the open (small $\kappa$ ) Hopf curves die at the same $D Z$-point on the divergence curve, 


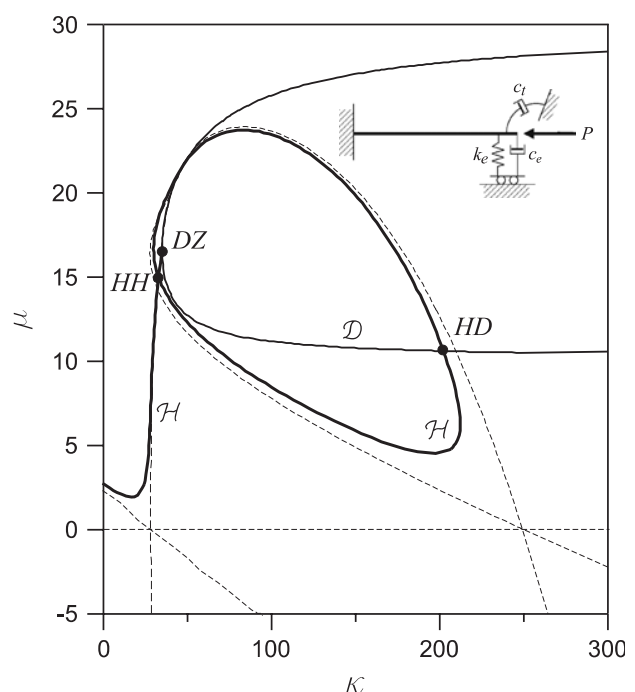

Fig. 5. Linear stability diagram for the torsionally damped system slightly perturbed by a small extensional damping; comparison with the unperturbed system; $\xi_{\mathrm{e}}=0.0025, \xi_{\mathrm{t}}=0.005$.
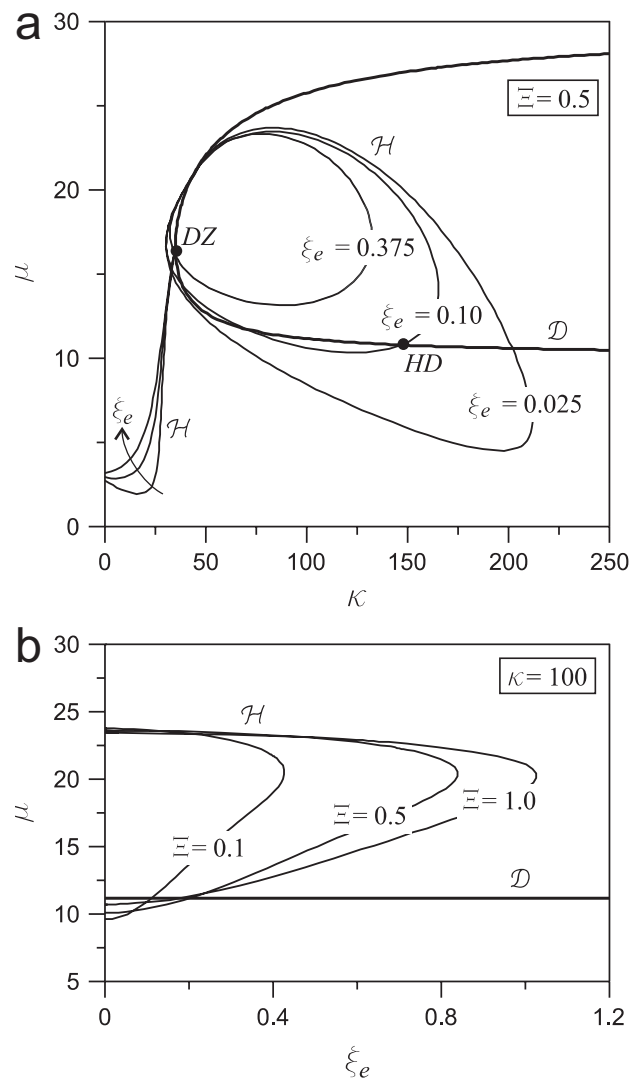

Fig. 6. Linear stability diagram for the complete model: (a) $\Xi=\xi_{\mathrm{e}} / \xi_{\mathrm{t}}=$ const; (b) $\kappa=$ const.

as already noted in literature for simple, discrete systems [31]. The close (larger $\kappa$ ) Hopf curves also intersect the $\mathscr{D}$-curve at different, damping-dependent, $\mathscr{H} \mathscr{D}$-points. The influence of the $\Xi$-parameter is shown in Fig. $6 \mathrm{~b}$, where $\kappa$ was kept fixed and $\Xi$ varied. It is observed that the range of $\xi_{\mathrm{e}}$, in which
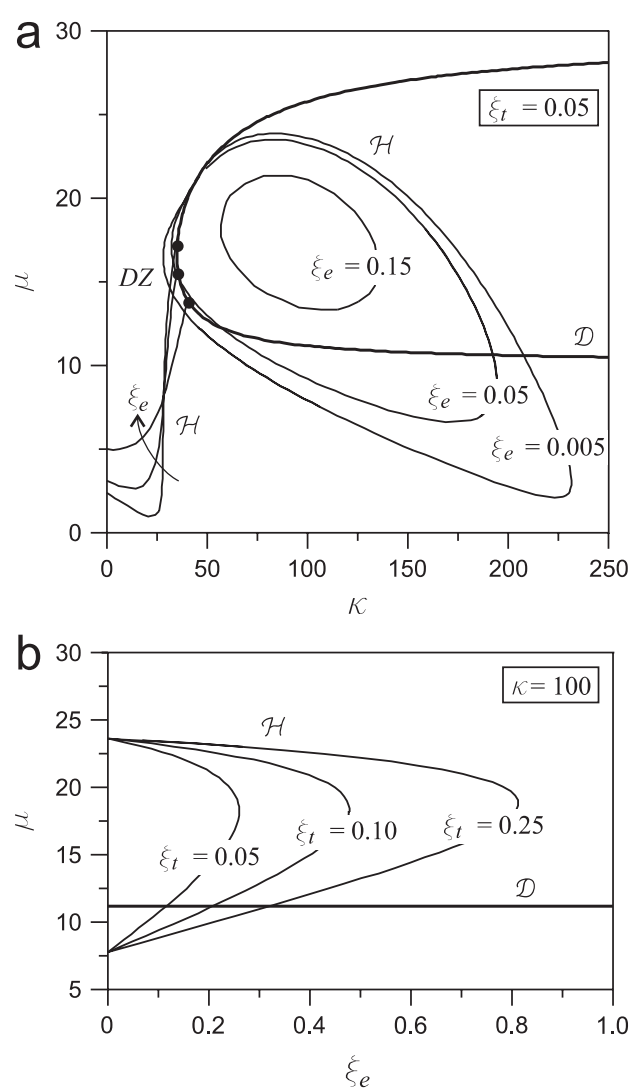

Fig. 7. Linear stability diagram for the complete model: (a) $\xi_{\mathrm{t}}=$ const and different $\xi_{\mathrm{e}}$ 's; (b) $\kappa=$ const and different $\xi_{\mathrm{t}}$ 's.

the dynamic bifurcation occurs at a load lower than the static bifurcation, sensibly depends on $\Xi$. In Fig. 7a only $\xi_{\mathrm{e}}$ was varied, by keeping $\xi_{\mathrm{t}}$ fixed. The property of coalescence of $D Z$-points does not hold any more (as for discrete systems); in addition, for large $\xi_{\mathrm{e}}$-damping, the close Hopf curve does not intersect the divergence curve. The analysis, when repeated for a fixed $\kappa$ and different values of the $\xi_{\mathrm{t}}$-parameter, leads to the results of Fig. 7b. This shows that, when $\xi_{\mathrm{e}}$ vanishes, the critical load is independent of $\xi_{\mathrm{t}}$, consistently with results of Fig. 4.

\section{Post-critical analysis}

The multiple scale method [32] is applied to analyze the system behavior at a $\mathscr{H} \mathscr{D}$ and at a non-resonant double-Hopf points. The fundamental steps of the method are resumed here. A perturbation parameter $\varepsilon$ is introduced as a measure of the distance of a generic point from a bifurcation point in the parameter space $(\kappa, \mu)$. Several independent time-scales $t_{k}:=$ $\varepsilon^{k} t$ are defined and the state variable $u(s)$ is expanded in a Taylor series of $\varepsilon$. By equating terms of the same power of $\varepsilon$, linear perturbation equations having the same operator are obtained, and then solved in sequence for the series coefficients. Except for the lower-order eigenvalue problem, higher-order equations are non-homogeneous; they admit a solution if and only if the known term belongs to the range of the singular operator, i.e. if it is orthogonal to the solutions of the adjoint 
homogeneous problem (13). Solvability equations furnish the bifurcation equations, governing the asymptotic dynamics of the system, reduced to the center manifold. The procedure is sketched ahead.

\subsection{Bifurcation equations}

The stiffness $\kappa$ and the load $\mu$ are taken as bifurcation parameters, and their increments with respect to the critical values $\kappa_{0}$ and $\mu_{0}$, denoted by, and ordered as

$\beta:=\kappa-\kappa_{0}=\mathcal{O}\left(\varepsilon^{2}\right), \quad \gamma:=\mu-\mu_{0}=\mathcal{O}\left(\varepsilon^{2}\right)$.

By introducing the series expansions:

$u=\varepsilon u_{1}+\varepsilon^{3} u_{3}+\cdots, \quad \frac{\mathrm{d}}{\mathrm{d} t}=d_{0}+\varepsilon^{2} d_{2}+\cdots$

with $d_{k}=\partial / \partial t_{k}$ and $t_{k}=\varepsilon^{k} t(k=0,2, \ldots)$, the following perturbation equations and boundary conditions are derived:

Order ع:

$\left\{\begin{array}{l}d_{0}^{2} u_{1}+u_{1}^{\prime \prime \prime}+2 \mu_{0} u_{1}^{\prime \prime}=0, \\ u_{1 A}=0, \quad u_{1 A}^{\prime}=0, \\ \xi_{\mathrm{e}} d_{0} u_{1 B}-u_{1 B}^{\prime \prime \prime}+\kappa_{0} u_{1 B}=0, \\ \xi_{\mathrm{t}} d_{0} u_{1 B}^{\prime}+u_{1 B}^{\prime \prime}=0 .\end{array}\right.$

Order $\varepsilon^{3}$ :

$\left\{\begin{array}{l}d_{0}^{2} u_{3}+u_{3}^{\prime \prime \prime \prime}+2 \mu_{0} u_{3}^{\prime \prime}=-2 d_{0} d_{2} u_{1}-2 \gamma u_{1}^{\prime \prime}-u_{1}^{\prime \prime}{ }^{3} \\ \quad-4 u_{1}^{\prime} u_{1}^{\prime \prime} u_{1}^{\prime \prime \prime}-u_{1}^{\prime 2} u_{1}^{\prime \prime \prime \prime} \\ \quad-\mu_{0}\left(3 u_{1}^{\prime 2}-u_{1 B}^{\prime 2}\right) u_{1}^{\prime \prime}-\left[\int_{1}^{s} d_{0}^{2}\left(\int_{0}^{s} \frac{1}{2} u_{1}^{\prime 2} \mathrm{~d} s\right) \mathrm{d} s\right] u_{1}^{\prime \prime} \\ \quad-\left[\left(\int_{0}^{s} d_{0}^{2} \frac{1}{2} u_{1}^{\prime 2} \mathrm{~d} s\right)\right] u_{1}^{\prime}, \\ u_{3 A}=0, \quad u_{3 A}^{\prime}=0, \\ \xi_{\mathrm{e}} d_{0} u_{3 B}-u_{3 B}^{\prime \prime \prime}+\kappa_{0} u_{3 B}=-\xi_{\mathrm{e}} d_{2} u_{1 B}-\beta u_{1 B} \\ \quad-\mu_{0} u_{1 B}^{\prime 3}+u_{1 B}^{\prime \prime \prime} u_{1 B}^{\prime 2}+u_{1 B}^{\prime \prime}{ }^{2} u_{1 B}^{\prime}, \\ \xi_{\mathrm{t}} d_{0} u_{3 B}^{\prime}+u_{3 B}^{\prime \prime}=-\xi_{\mathrm{t}} d_{2} u_{1 B}^{\prime}-\frac{1}{2} \xi_{\mathrm{t}} u_{1 B}^{\prime 2} d_{0} u_{1 B}^{\prime}-\frac{1}{2} u_{1 B}^{\prime \prime} u_{1 B}^{\prime}{ }^{2} .\end{array}\right.$

Eqs. (14) (generating solution) admit the following nondecaying solution:

$$
\begin{aligned}
u_{1}= & A_{1}\left(t_{2}, t_{4}, \ldots\right) \phi_{1}(s) \mathrm{e}^{\mathrm{i} \omega_{1} t_{0}}+A_{2}\left(t_{2}, t_{4}, \ldots\right) \phi_{2}(s) \mathrm{e}^{\mathrm{i} \omega_{2} t_{0}} \\
& + \text { c.c. }
\end{aligned}
$$

or

$u_{1}=a_{1}\left(t_{2}, t_{4}, \ldots\right) \phi_{1}(s)+A_{2}\left(t_{2}, t_{4}, \ldots\right) \phi_{2}(s) \mathrm{e}^{\mathrm{i} \omega t_{0}}+$ c.c.

for non-resonant double Hopf or $\mathscr{H} \mathscr{D}$ bifurcation, respectively. In Eqs. (15) $A_{j}=\frac{1}{2} a_{j} \exp \left(i \theta_{j}\right)(j=1,2)$ are complex constants with real amplitudes $a_{j}$ and phases $\theta_{j}, \phi_{j}(s)$ are complex eigenfunctions and c.c. stands for the complex conjugate of preceding terms. The same notation holds for Eq. (16), with $a_{1} \in \mathbb{R}$ and $\phi_{1}(s) \in \mathbb{R}$. Substituting Eqs. (15) or (16) in Eqs. (141) and enforcing solvability, bifurcation equations, formally identical for the two cases, are drawn:

$$
\left\{\begin{aligned}
\dot{a}_{1}= & \left(\alpha_{1 \beta} \beta+\alpha_{1 \gamma} \gamma\right) a_{1}+R_{122} a_{1} a_{2}^{2}+R_{111} a_{1}^{3} \\
& +\mathcal{O}\left(\left|a_{1}\right|^{5}+\left|a_{2}\right|^{5}\right), \\
\dot{a}_{2}= & \left(\alpha_{2 \beta} \beta+\alpha_{2 \gamma} \gamma\right) a_{2}+R_{112} a_{1}^{2} a_{2}+R_{222} a_{2}^{3} \\
& +\mathcal{O}\left(\left|a_{1}\right|^{5}+\left|a_{2}\right|^{5}\right) .
\end{aligned}\right.
$$

The coefficients $\alpha$ 's and R's appearing in Eqs. (17) was obtained via a symbolic manipulation program [33]. Since they assume quite cumbersome forms, they are not reported here. However, the numerical values that they assume at the bifurcation points studied ahead (Section 4.2), are given in the Appendix C. Eqs. (17) constitute the bifurcation equations, in standard normal form and specialized to a symmetric system, for two pairs of non-resonant purely imaginary critical eigenvalues, or for a one zero and a purely imaginary pair of critical eigenvalues [11]. They are invariant under the transformations $a_{1} \rightarrow-a_{1}$ and/or $a_{2} \rightarrow-a_{2}$.

In order to build-up the bifurcation diagram, it is first necessary to evaluate the steady-state solutions of the dynamical system (17) and, then, to perform a stability analysis. The fixed points of Eqs. (17) are determined by setting $\dot{a}_{1}=\dot{a}_{2}=0$. These solutions, in terms of the original system, correspond to two-frequency quasi-periodic motions around the rectilinear configuration of the beam or to one-frequency periodic motion around a non-trivial (buckled) position, respectively. Eqs. (17) admit the trivial solution $a_{1 T}=a_{2 T}=0$. Non-trivial steady-state solutions, with one or two non-vanishing components are then sought. If $a_{2}=0$, Eq. $\left(17_{2}\right)$ is identically satisfied, while Eq. $\left(17_{1}\right)$ yields:

$a_{1}^{2}=-\frac{\alpha_{1 \beta} \beta+\alpha_{1 \gamma \gamma}}{R_{111}}$.

Similarly, if $a_{1}=0$, Eq. (17 $)$ is identically satisfied, while Eq. $\left(17_{2}\right)$ yields:

$a_{2}^{2}=-\frac{\alpha_{2 \beta} \beta+\alpha_{2 \gamma} \gamma}{R_{222}}$.

If both $a_{1}$ and $a_{2}$ are different from zero, Eqs. (17) give

$a_{1}^{2}=\frac{\left(\alpha_{2 \beta} R_{221}-\alpha_{1 \beta} R_{222}\right) \beta+\left(\alpha_{2 \gamma} R_{221}-\alpha_{1 \gamma} R_{222}\right) \gamma}{R_{111} R_{222}-R_{221} R_{211}}$,

$a_{2}^{2}=\frac{\left(\alpha_{2 \beta} R_{111}-\alpha_{1 \beta} R_{211}\right) \beta+\left(\alpha_{2 \gamma} R_{111}-\alpha_{1 \gamma} R_{211}\right) \gamma}{R_{221} R_{211}-R_{111} R_{222}}$.

Since $a_{1}$ and $a_{2}$ are real, solutions (18)-(20) exist only in a sub-domain of the control parameter plane. Finally, stability of the fixed points is analyzed by evaluating the eigenvalues of the Jacobian matrix at equilibria.

\subsection{Numerical results}

The reduced dynamical system (17) is numerically analyzed for two sample systems, respectively at a $\mathscr{H} \mathscr{H}$ and at $\mathscr{H} \mathscr{D}$ points. Fig. 8 illustrates the $\mathscr{H} \mathscr{H}$ and $\mathscr{H} \mathscr{D}$ bifurcation points, the relevant critical parameters $\kappa_{0}$ and $\mu_{0}$. 


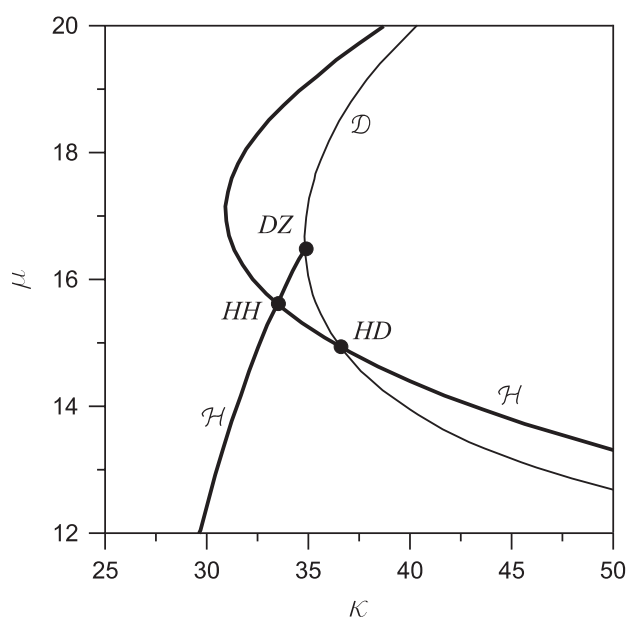

Fig. 8. Codimension-two bifurcation points: Hopf-Hopf ( $\mathscr{H} \mathscr{H}$ ) and Hopfdivergence $(\mathscr{H} \mathscr{D}) ; \xi_{\mathrm{t}}=0.250, \xi_{\mathrm{e}}=0.125 ; \mathscr{H} \mathscr{H}:\left(\kappa_{0}=33.478, \mu_{0}=15.602\right)$; $\mathscr{H} \mathscr{D}:\left(\kappa_{0}=36.569, \mu_{0}=14.926\right)$.

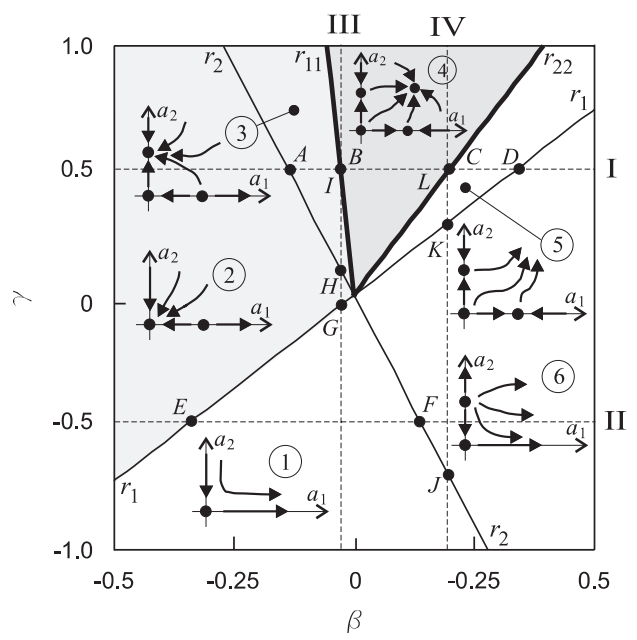

Fig. 9. Bifurcation diagram and phase-portraits for the $\mathscr{H} \mathscr{H}$ bifurcation.

The $\mathscr{H} \mathscr{H}$ bifurcation is studied first. The bifurcation diagram is shown in Fig. 9. The parameter plane is divided into six sectors, delimited by four straight lines passing for the origin. Lines $r_{1}$ and $r_{2}$, individually delimit the half-plane in which monomodal periodic solutions $\left(a_{1}, 0\right)$ and $\left(0, a_{2}\right)$, respectively, exist, according to Eqs. (18) and (19); lines $r_{11}$ and $r_{22}$, together, delimit the sector in which the bimodal quasi-periodic solution $\left(a_{1}, a_{2}\right)$ exists, according to Eqs. (20). A sketch of the phase portraits relevant to the six sectors is also shown in the figure. It appears that stable solutions only exist in sectors 2 (trivial solution), 3 , where the motion is periodic, and in sector 4 , where the motion is bi-periodic.

Some equilibrium paths, denoted by I to IV in Fig. 9, are shown in Fig. 10. They can be thought as planar sections off three-dimensional plots $\left(a_{i}, \beta, \gamma\right)$, with $i=1,2$. Points $A$ to $L$ marked in Fig. 10 correspond to homonymous points in Fig. 9. It is seen that (Fig. 10a), for $\beta$ increasing along path $\mathrm{I}$, the trivial solution is (locally) stable up to point $A$, where a stable
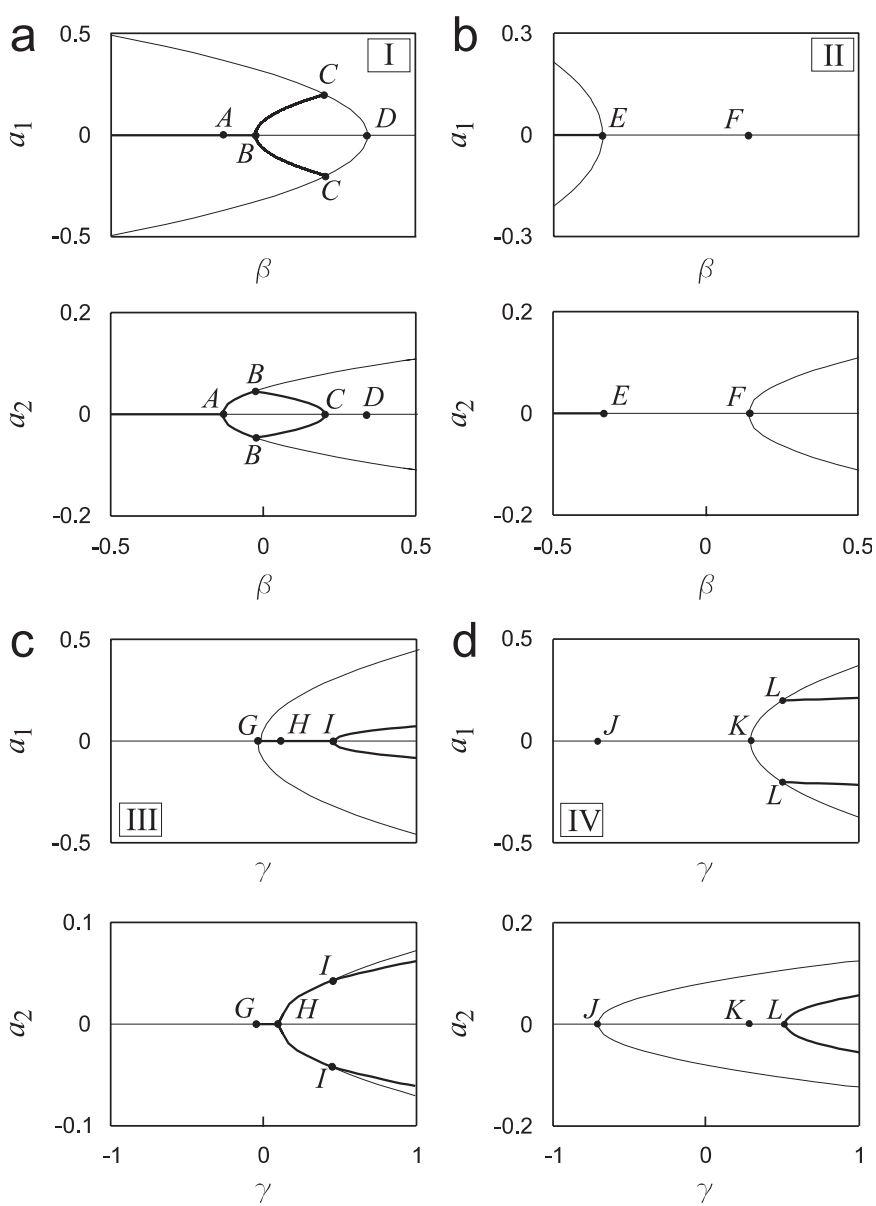

Fig. 10. Equilibrium paths for the $\mathscr{H} \mathscr{H}$ bifurcation.

monomodal $a_{2}$-motion takes place. Then, at point $B$, such motion exchanges stability with a bimodal $\left(a_{1}, a_{2}\right)$-motion. This disappears at point $C$, where only an unstable $a_{1}$-motion, originating at $D$, exists. Behind $D$, only the unstable $a_{2}$-motion, originated at $A$, exists. Fig. $10 \mathrm{~b}$, relevant to path II, is selfexplaining. Fig. 10c illustrates path III. At point $G$ un unstable $a_{1}$-motion arises; at point $H$ a stable $a_{2}$-motion takes place. This exchange stability at $I$ with the stable $\left(a_{1}, a_{2}\right)$-motion. Path IV is finally shown in Fig. 10d, that displays as the $a_{1}$-amplitude of the bi-periodic motion does not depend on the load $\gamma=\mu-\mu_{0}$.

The $\mathscr{H} \mathscr{D}$ bifurcation is then addressed. The relevant bifurcation diagram is plotted in Fig. 11. Here, lines $r_{1}, r_{2}, r_{11}$ and $r_{22}$ keep the meaning previously illustrated, where the index 1 refers to divergence and index 2 to Hopf. As a peculiarity of this system, lines $r_{2}$ and $r_{22}$ are nearly undistinguishable; as a result, the parameter plane is divided in only five sectors. The relevant phase-portraits, in contrast with the previous case (Fig. 9), reveal the existence of a stable solution in the whole plane. The two equilibrium paths shown in Fig. 12 are therefore sufficient to illustrate the post-critical behavior. Along path I (Fig. 12a), a divergence of amplitude $a_{1}$ occurs at point $A$. Then, at point $B$, two bifurcations coalesce: an unstable periodic $a_{2}$-motion and a new, stable, two-components motion $\left(a_{1}, a_{2}\right)$. Along path II (Fig. 12b), a stable periodic motion around the 


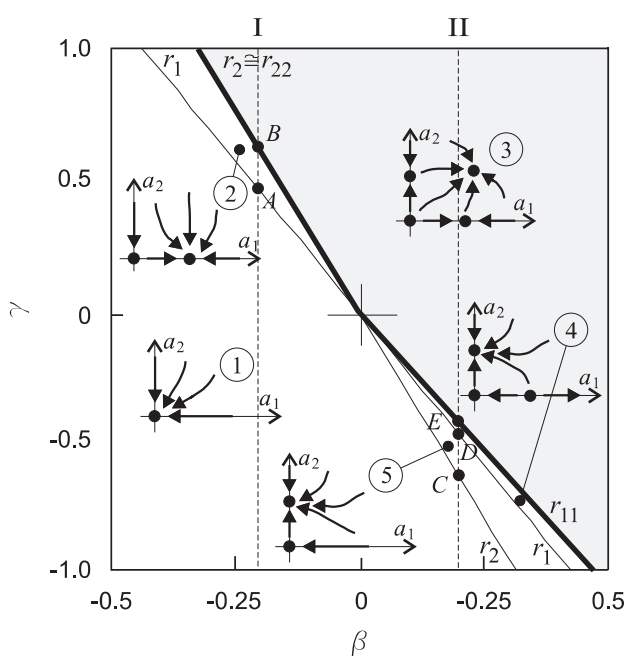

Fig. 11. Bifurcation diagram and phase-portraits for the $\mathscr{H} \mathscr{D}$ bifurcation.
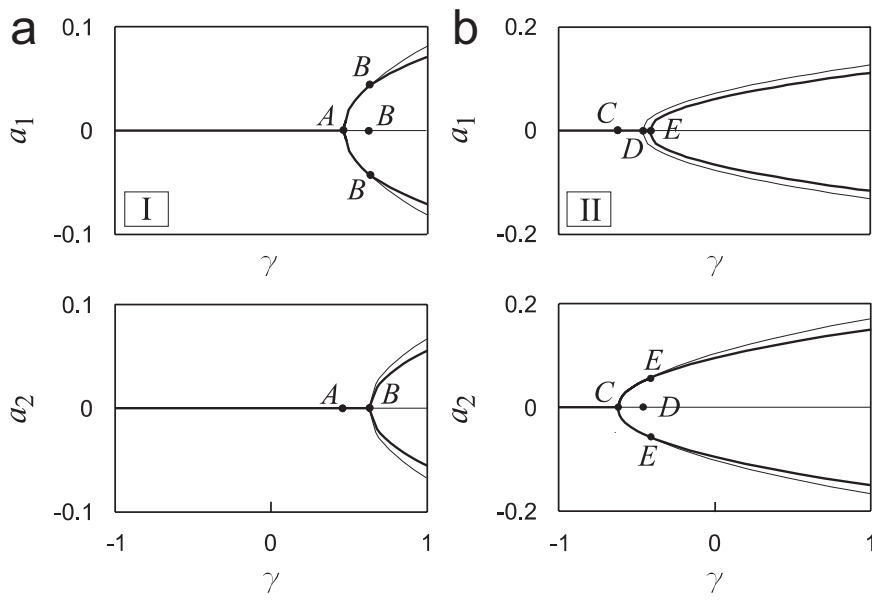

Fig. 12. Equilibrium paths for the $\mathscr{H} \mathscr{D}$ bifurcation.

trivial configuration is triggered $C$; then, at $D$, after an unstable static bifurcation, a new equilibrium path arises. Finally, at point $E$, the motion around the buckled configuration loses stability, and a stable $\left(a_{1}, a_{2}\right)$ periodic motion around a new equilibrium position takes place.

\section{Conclusions}

The critical and post-critical behavior of a beam constrained by lumped spring and dashpots, loaded by a follower force, has been studied. The following conclusions are drawn.

1. The beam undergoes Hopf, divergence, double-zero, Hopf-divergence and double-Hopf bifurcations, resonant or not, for different combinations of parameters.

2. If both dashpots are removed, an Hamiltonian Hopf bifurcation takes place along a curve tangent to the divergence curve, similarly to known results.

3. If only a dashpot of extensional type is added to the undamped beam, however small is the damping coefficient, it has a destabilization effect for any values of the spring stiffness, and the well-known Ziegler paradox takes place. Discontinuity exists between the undamped and the damped system.

4. If only a torsional damper is added to the undamped beam, an infinite number of resonant double-Hopf manifests itself for zero values of the load, so that simple Hopf bifurcations occur both for positive (compressive) and negative (tensile) forces. However, if a small extensional damping is added, such bifurcations are destroyed and instability for traction disappears.

5. In the purely torsional damping case, the critical mode at a generic Hopf point entails vanishing of both bending and rotation at the tip of the beam. Therefore, as a paradox, the non-conservative force behaves as a conservative one at the bifurcation.

6. A non-degenerate double-zero bifurcation, occurring at the crossing of a Hopf and a divergence curve, where the first one dies, only can exist if both dampers are present in the structure.

7. The scenario of the complete model can usefully be interpreted as a perturbation, due to the extensional damping, of the scenario of the purely torsionally damped structure.

8. The bifurcation equations relevant to the non-resonant double-Hopf and Hopf-divergence bifurcations are formally similar. They lead to a bifurcation scenario in a two-dimensional parameter space in which periodic motions around the trivial or buckled configurations, as well as bi-periodic motions, are present. Some sections of the amplitude-parameter surfaces have been given.

\section{Acknowledgments}

This work was partially supported under the FY 2005-2007 PRIN Grant 'Modelling and experimental tests of the dynamic behavior of flexible structures' from the Italian Ministry of Education, University and Scientific Research.

\section{Appendix A. Equations of motion}

A Lagrangian description of the motion is adopted (Fig. 1b). The beam is straight in its rest reference configuration $\mathscr{C}_{0}$, coincident with the prestressed equilibrium state, with directors $\mathbf{D}_{j},(j=x, y)$, oriented as the orthonormal versors of a fixed inertial reference frame $\left(A ; \mathbf{a}_{j}\right)$. The reference configuration is therefore described by $\mathbf{X}(s)=s \mathbf{a}_{x}$ where $s \in(0, l)$ denotes an abscissa and $l$ is the beam length. The referential description of the current beam configuration $\mathscr{C}_{\mathrm{t}}$ at time $t$ is specified by the position vector $\mathbf{x}(s, t)=\mathbf{X}(s)+\mathbf{u}(s, t)$ with $\mathbf{u}(s, t)$ the vector displacement, and by the proper orthogonal tensor $\mathbf{R}(s, t)$ describing the rotation that leads the directors $\mathbf{D}_{j}$ to match the current directors $\mathbf{d}_{j}$ (i.e. $\mathbf{d}_{j}=\mathbf{R} \mathbf{D}_{j}$ ); in the fixed inertial reference $\left(A ; \mathbf{a}_{j}\right)$, it admits the following scalar representation:

$\mathbf{R}=\left(\begin{array}{cc}\cos \vartheta & -\sin \vartheta \\ \sin \vartheta & \cos \vartheta\end{array}\right)$ 
where $\vartheta$ is the amplitude rotation around the axes oriented by the vector $\mathbf{a}_{z}=\mathbf{a}_{x} \times \mathbf{a}_{y}$.

Denoting by $\left(u_{x}, u_{y}\right)$ the Cartesian components of $\mathbf{u}$ in the basis $\left(\mathbf{a}_{x}, \mathbf{a}_{y}\right)$, the current configuration $\mathscr{C}_{\mathrm{t}}$ is described by the longitudinal displacement $u_{x}(s, t)$, the transversal displacement field $u_{y}(s, t)$ and the rotation $\vartheta(s, t)$. The three displacements, however, are not independent, because of the internal constraints:

$\tan \vartheta=\frac{u_{y}^{\prime}}{1+u_{x}^{\prime}}, \quad\left(1+u_{x}^{\prime}\right)^{2}+u_{y}^{\prime 2}-1=0$

expressing shear-undeformability and inextensibility, respectively (where $(\bullet)^{\prime}:=\mathrm{d} / \mathrm{d} s$ ). By taking $u:=u_{y}$ as active kinematic variable, from Eqs. (22), the remaining passive variables $\left(u_{x}, \vartheta\right)$, truncated at the third order, are obtained:

$u_{x}=-\frac{1}{2} \int_{0}^{s} u^{\prime 2} \mathrm{~d} x, \quad \vartheta=u^{\prime}+\frac{1}{6} u^{\prime 3}$,

where the geometrical boundary conditions,

$u_{x}(0, t)=0, \quad \vartheta(0, t)=0$

have been used. Furthermore, the unique non-zero strain measure, the curvature $\chi(s, t):=\vartheta^{\prime}$, becomes

$\chi=u^{\prime \prime}+\frac{1}{2} u^{\prime 2} u^{\prime \prime}$.

Denoting by $\mathbf{t}(s, t)$ and $\mathbf{m}(s, t)$ the internal contact force and couple, respectively, and $\mathbf{b}(s, t)$ and $\mathbf{c}(s, t)$ the distributed body forces and couples per unit reference length, respectively, the dynamic balance equations, in Lagrangian form, are expressed as

$\mathbf{t}^{\prime}+\mathbf{b}=\mathbf{0}, \quad \mathbf{m}^{\prime}+\mathbf{x}^{\prime} \times \mathbf{t}+\mathbf{c}=\mathbf{0}$

along with the mechanical boundary conditions:

$\mathbf{t}_{B}=-\left(k_{\mathrm{e}} u_{B}+c_{\mathrm{e}} \dot{u}_{B}\right) \mathbf{a}_{y}-P \mathbf{d}_{B}, \quad \mathbf{m}_{B}=-c_{\mathrm{t}} \dot{\vartheta}_{B} \mathbf{a}_{z}$

where $B$ denotes evaluation at the end $B$ of the beam and the overdot indicates differentiation with respect to time. Equilibrium in $\mathscr{C}_{0}$ entails that $\mathbf{t}_{0}(s)=-P \mathbf{a}_{x}$ and $\mathbf{m}_{0}(s)=\mathbf{0}$. To obtain the scalar form of the incremental balance equations, the internal contact forces and couples in the current configuration $\mathscr{C}_{\mathrm{t}}$ are expressed as $\mathbf{t}(s, t)=[-P+H(s, t)] \mathbf{a}_{x}+V(s, t) \mathbf{a}_{y}$ and $\mathbf{m}(s, t)=M(s, t) \mathbf{a}_{z}$. Recalling that, for Kirchhoff's beam model, $\mathbf{x}^{\prime}=\mathbf{d}_{x}=\cos \vartheta \mathbf{a}_{x}+\sin \vartheta \mathbf{a}_{y}$, the equations of motions (26) for the dynamic components, projected onto $\left(\mathbf{a}_{x}, \mathbf{a}_{y}\right)$-basis, are

$H^{\prime}-m \ddot{u}_{x}=0, \quad V^{\prime}-m \ddot{u}=0$,

$M^{\prime}+V \cos \vartheta+(P-H) \sin \vartheta=0$

while the boundary conditions Eq. (27) becomes

$H_{B}=P\left(1-\cos \vartheta_{B}\right), \quad V_{B}=-\left(k_{\mathrm{e}} u_{B}+c_{\mathrm{e}} \dot{u}_{B}\right)-P \sin \vartheta_{B}$,

$M_{B}=-c_{\mathrm{t}} \dot{\vartheta}_{B}$

having neglected the rotatory inertia and expressed the translational inertial forces in the form $\mathbf{b}=-m\left(\ddot{u}_{x} \mathbf{a}_{x}+\ddot{u} \mathbf{a}_{y}\right)$, where $m$ is the mass density.
In Eqs. (28), $H$ and $V$ are reactive internal forces. In order to express the equilibrium equations in terms of the unique configuration variable $u$, they must be condensed. To this end, from Eq. (281) the component $H$ is derived, namely

$H=P\left(1-\cos \vartheta_{B}\right)+\int_{l}^{s} m \ddot{u}_{x} \mathrm{~d} s$,

where the boundary condition Eq. $\left(29_{1}\right)$ has been used. To eliminate the $V$ component Eq. (283) is solved with respect to $V$, thereby obtaining $V=-M^{\prime} / \cos \vartheta+(H-P) \tan \vartheta$. Then, substituting $V$ into Eq. (282) it follows:

$m \ddot{u}+\left(\frac{M^{\prime}}{\cos \vartheta}\right)^{\prime}+[(P-H) \tan \vartheta]^{\prime}=0$

while the boundary conditions Eq. $\left(29_{2,3}\right)$ becomes

$M_{B}^{\prime}=-\left(k_{\mathrm{e}} u_{B}+c_{\mathrm{e}} \dot{u}_{B}\right) \cos \vartheta_{B}, \quad M_{B}=-c_{\mathrm{t}} \dot{\vartheta}_{B}$.

A linear constitutive equation is assumed in the form $M=$ $E I \chi$, where $E I$ is the flexural stiffness of the beam. Substituting it in Eq. (31), using Eq. (22), and Taylor expanding up-to third order, the following equation is obtained:

$$
\begin{aligned}
m \ddot{u} & +E I u^{\prime \prime \prime \prime}+P u^{\prime \prime}+E I\left(u^{\prime \prime 3}+4 u^{\prime} u^{\prime \prime} u^{\prime \prime \prime}+u^{\prime 2} u^{\prime \prime \prime \prime}\right) \\
& +\frac{1}{2} P\left(3 u^{\prime 2}-u_{B}^{\prime 2}\right) u^{\prime \prime}+\left[m \int_{l}^{s}\left(\int_{0}^{s} \frac{1}{2} u^{\prime 2} \mathrm{~d} s\right)^{\cdot \cdot} \mathrm{d} s\right] u^{\prime \prime} \\
& +\left[m\left(\int_{0}^{s} \frac{1}{2} u^{\prime 2} \mathrm{~d} s\right)^{\cdot \cdot}\right] u^{\prime}=0
\end{aligned}
$$

while the associated kinematic and dynamic boundary conditions becomes:

$$
\begin{aligned}
& u_{A}=0, \quad u_{A}^{\prime}=0, \\
& -E I u_{B}^{\prime \prime \prime}+k_{\mathrm{e}} u_{B}+c_{\mathrm{e}} \dot{u}_{B}=-\frac{1}{2} P u_{B}^{\prime 3}+E I\left(u_{B}^{\prime \prime \prime} u_{B}^{\prime 2}+u_{B}^{\prime \prime 2} u_{B}^{\prime}\right), \\
& E I u_{B}^{\prime \prime}+c_{\mathrm{t}} \dot{u}_{B}^{\prime}=-\frac{1}{2} c_{\mathrm{t}} u_{B}^{\prime 2} \dot{u}_{B}^{\prime}-\frac{1}{2} E I u_{B}^{\prime \prime} u_{B}^{\prime 2} .
\end{aligned}
$$

When Eqs. (33) and (34) are put in nondimensional form via Eqs. (3), Eqs. (1) and (2) are finally derived.

\section{Appendix B. Eigenvalue transversality condition}

Let $O \equiv\left(\kappa_{0}, \mu_{0}\right)$ a point of the parameter space at which the eigenvalue $\lambda$ is purely imaginary, namely $\lambda_{0}:=\mathrm{i} \omega_{0}$ (Hopfpoint). In order the bifurcation occurs, however, $\mathfrak{R}[\partial \lambda / \partial \varepsilon] \neq 0$, with $\varepsilon$ a perturbation of the parameters (not tangent to $\mathscr{H}$ ). By letting

$\lambda=\lambda_{0}+\varepsilon \lambda_{1}+\cdots, \quad \mathbf{c}=\mathbf{c}_{0}+\varepsilon \mathbf{c}_{1}+\cdots$

in the eigenvalue problem $\mathbf{S}(\lambda ; \varepsilon) \mathbf{c}(\varepsilon)=\mathbf{0}$, the perturbation equations follow (see also [34]):

$\varepsilon^{0}: \mathbf{S}^{0} \mathbf{c}_{0}=\mathbf{0}$,

$\varepsilon: \mathbf{S}^{0} \mathbf{c}_{1}=\left[\mathbf{S},{ }_{\varepsilon}^{0}+\mathbf{S},{ }_{\lambda}^{0} \lambda_{1}\right] \mathbf{c}_{0}$, 
where a comma denotes total differentiation with respect the following variable, and the apex 0 denotes evaluation at $C$. Enforcing solvability of the $\varepsilon$-order equation, it follows:

$\lambda_{1}=-\frac{\mathbf{b}_{0}^{\mathrm{H}} \mathbf{S},{ }_{\varepsilon}^{0} \mathbf{c}_{0}}{\mathbf{b}_{0}^{\mathrm{H}} \mathbf{S},{ }_{\lambda}^{0} \mathbf{c}_{0}}$

where $\mathbf{b}_{0}$ is the left eigenvector of $\mathbf{S}^{0}$ (namely, $\left(\mathbf{S}^{0}\right)^{\mathrm{H}} \mathbf{b}_{0}=\mathbf{0}$, with $\mathrm{H}$ the transpose conjugate). Eq. (37) represents the sensitivity of $\lambda$ at $O$; if $\mathfrak{R}\left[\lambda_{1}\right] \neq 0$, then transversality is satisfied.

\section{Appendix C. Coefficients of the bifurcation equations}

The numerical values assumed by the critical parameters, the eigenpairs and the coefficients appearing in the bifurcation equations (17) are reported here for the two selected points, $\mathscr{H} \mathscr{H}$ and $\mathscr{H} \mathscr{D}$ of Fig. 8.

\section{C.1. Double Hopf}

The critical values of the parameters are $\kappa_{0}=33.478, \mu_{0}=$ $15.602, \xi_{\mathrm{t}}=0.250, \xi_{\mathrm{e}}=0.125$. The frequencies $\omega_{i}(i=1,2)$ associated with the two interactive modes, and the coefficients $p, q, c_{1}$ and $c_{2}$ appearing in the relevant (right) critical eigenvectors $\phi_{i}(s)$ (Eqs. (6) and (7)) are given in Table 1. The values assumed by coefficients $c_{1}$ and $c_{2}$ in the associated left eigenvectors $\psi_{i}(s)$ (still given by Eq. (7)), are also given in Table 1. The complex bifurcation equations read:

$\dot{A}_{1}=\left(C_{1 \beta} \beta+C_{1 \gamma} \gamma\right) A_{1}+C_{111} A_{1}^{2} \bar{A}_{1}+C_{122} A_{1} A_{2} \bar{A}_{2}$

$\dot{A}_{2}=\left(C_{2 \beta} \beta+C_{2 \gamma} \gamma\right) A_{2}+C_{112} A_{1} \bar{A}_{1} A_{2}+C_{222} A_{2}^{2} \bar{A}_{2}$

with coefficients reported in Table 2 . In Eq. (17), $\alpha_{i \beta}=\mathfrak{R}\left[C_{i \beta}\right]$, $\alpha_{i \gamma}=\mathfrak{R}\left[C_{i \gamma}\right], R_{i j k}=\mathfrak{R}\left[C_{i j k}\right]$, while the imaginary parts affect the frequency corrections, not investigated here.

\section{C.2. Hopf-divergence}

The critical values of the parameters are $\kappa_{0}=36.569, \mu_{0}=$ $14.926, \xi_{\mathrm{t}}=0.250, \xi_{\mathrm{e}}=0.125$. The frequencies $\omega_{i}(i=1,2)$ of the two modes and the parameters $p$ and $q$ are reported in Table 3. Concerning the eigenvectors, $\phi_{1}(s)$ assumes the following form when indeterminacy has been resolved:

$\phi_{1}(s)=c_{1}(\cos p x-1)+c_{2}(\sin p x-p x)$

Table 1

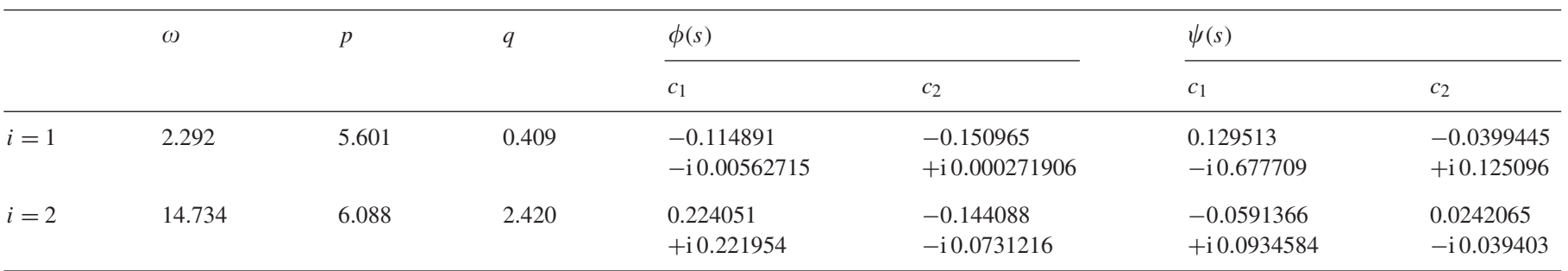

Table 2

\begin{tabular}{ll}
\hline$C_{1 \beta}=0.316801-\mathrm{i} 0.454693$ & $C_{2 \beta}=0.243336-\mathrm{i} 1.48525$ \\
$C_{1 \gamma}=-0.215225-\mathrm{i} 0.590027$ & $C_{2 \gamma}=0.0672233+\mathrm{i} 0.122567$ \\
$C_{111}=4.44357+\mathrm{i} 86.2402$ & $C_{112}=-8.32264+\mathrm{i} 152.819$ \\
$C_{122}=228.457+\mathrm{i} 1041.15$ & $C_{222}=-52.8691-\mathrm{i} 69.6809$
\end{tabular}

Table 3

\begin{tabular}{|c|c|c|c|c|c|c|c|}
\hline & \multirow[t]{2}{*}{$\omega$} & \multirow[t]{2}{*}{$p$} & \multirow[t]{2}{*}{$q$} & \multicolumn{2}{|l|}{$\phi(s)$} & \multicolumn{2}{|l|}{$\psi(s)$} \\
\hline & & & & $c_{1}$ & $c_{2}$ & $c_{1}$ & $c_{2}$ \\
\hline$i=1$ & 0 & 5.464 & 0 & -0.163872 & -0.153036 & -1.57691 & 0.288619 \\
\hline$i=2$ & 16.042 & 6.069 & 2.643 & $\begin{array}{l}0.274611 \\
+\mathrm{i} 0.232822\end{array}$ & $\begin{array}{l}-0.164199 \\
-\mathrm{i} 0.0871023\end{array}$ & $\begin{array}{l}-0.0418632 \\
+\mathrm{i} 0.0782989\end{array}$ & $\begin{array}{l}0.0186516 \\
-\mathrm{i} 0.035856\end{array}$ \\
\hline
\end{tabular}


and analogously for $\psi_{1}(s)$; Eq. (7) still holds for $\phi_{2}(s)$ and $\psi_{2}(s)$. The relevant coefficient $c_{1}$ and $c_{2}$ are displayed in Table 3. The complex bifurcation equations are

$\dot{A}_{1}=\left(C_{1 \beta} \beta+C_{1 \gamma} \gamma\right) A_{1}+C_{111} A_{1}^{3}+C_{122} A_{1} A_{2} \bar{A}_{2}$,

$\dot{A}_{2}=\left(C_{2 \beta} \beta+C_{2 \gamma} \gamma\right) A_{2}+C_{112} A_{1}^{2} A_{2}+C_{222} A_{2}^{2} \bar{A}_{2}$

with coefficient given in Table 4 . The same notation adopted for the double-Hopf holds for the coefficients $\alpha_{i \beta}, \alpha_{i \gamma}$ and $R_{i j k}$ of Eq. (17).

\section{References}

[1] M. Beck, Die Knicklast des einseiting eingespannten tangential gedrückten Stabes, Z. Angew. Math. Phys. 3 (1952) 225-228.

[2] B.R. Ryu, K. Katayama, Y. Sugiyama, Dynamic stability of Timoshenko columns subjected to subtangential forces, Comput. Struct. 68 (1998) 499-512.

[3] S.B. Andersen, J.J. Thomsen, Post-critical behavior of Beck's column with a tip mass, Int. J. Nonlinear Mech. 37 (2002) 135-151.

[4] F.M. Detinko, Lumped damping and stability of Beck column with a tip mass, Int. J. Solids Struct. 40 (2003) 4479-4486.

[5] O.N. Kirillov, A.P. Seyranian, Dissipation induced instabilities in continuous non-conservative systems PAMM Proc. Appl. Math. Mech., vol. 5, 2005, pp. 87-98. DOI 10.1002/pamm.200510029.

[6] O.N. Kirillov, A.P. Seyranian, The effect of small internal and external damping on the stability of distributed non-conservative systems, J. Appl. Math. Mech. 69 (2005) 529-552.

[7] A.N. Kounadis, On the failure of static stability analyses of nonconservative systems in regions of divergence instability, Int. J. Solids Struct. 31 (15) (1994) 2099-2120.

[8] A.N. Kounadis, Non-potential dissipative systems exhibiting periodic attractors in region of divergence, Chaos, Solitons and Fractals 8 (4) (1997) 583-612.

[9] V.I. Arnold, Geometrical Methods in the Theory of Ordinary Differential Equations, Springer, New York, Heidelberg, Berlin, 1982 (Russian original, Moscow, 1977).

[10] J. Guckenheimer, P. Holmes, Nonlinear Oscillations, Dynamical Systems, and Bifurcations of Vector Fields, Springer, New York, 1983

[11] H. Troger, A. Steindl, Nonlinear Stability and Bifurcation Theory, Springer, Wien, New York, 1991

[12] A. Steindl, H. Troger, Methods for dimension reduction and their application in non-linear dynamics, Int. J. Solids Struct. 38 (2001) 2147-3131.

[13] A. Luongo, A. Paolone, Perturbation methods for bifurcation analysis from multiple nonresonant complex eigenvalues, Nonlinear Dyn. 14 (1997) 193-210.

[14] A. Luongo, A. Paolone, Multiple scale analysis for divergence-Hopf bifurcation of imperfect symmetric systems, J. Sound Vib. 218 (1998) $527-539$.
[15] A. Luongo, A. Paolone, A. Di Egidio, Multiple time scales analysis for $1: 2$ and 1:3 resonant Hopf bifurcations, Nonlinear Dyn. 34 (3-4) (2003) 269-281.

[16] A. Luongo, A. Di Egidio, A. Paolone, Multiple time scale analysis for bifurcation from a multiple-zero eigenvalue, AIAA J. 41 (6) (2003) 1143-1150.

[17] A. Luongo, A. Di Egidio, A. Paolone, Multiscale analysis of defective multiple-Hopf bifurcations, Comput. Struct. 82 (2004) 2705-2722.

[18] A. Luongo, A. Di Egidio, A. Paolone, Multiple scale bifurcation analysis for finite-dimensional autonomous systems, in: Recent Research Developments in Sound and Vibration, vol. 1, Transworld Research Network, Kerala, India, ISBN:81-7895-031-6 161-201, 2002.

[19] A. Luongo, A. Di Egidio, Divergence, Hopf and double-zero bifurcations of a non-linear planar beam, Nonlinear Dyn. 41 (1-3) (2005) 171-190.

[20] A. Luongo, A. Di Egidio, Bifurcation equations through multiple-scales analysis for a continuous model of a planar beam, Comput. Struct. 84 (2006) 1596-1605.

[21] A. Paolone, M. Vasta, A. Luongo, Flexural-torsional bifurcations of a cantilever beam under potential and circulatory forces: I Nonlinear model and stability analysis, Int. J. Nonlinear Dyn. 41 (2006) 586-594.

[22] A. Paolone, M. Vasta, A. Luongo, Flexural-torsional bifurcations of a cantilever beam under potential and circulatory forces: II Post-critical analysis, Int. J. Nonlinear Dyn. 41 (2006) 595-604.

[23] A.H. Nayfeh, Reduced-order models of weakly non-linear spatially continuous systems, Nonlinear Dyn. 16 (1998) 105-125.

[24] A.H. Nayfeh, W. Lacarbonara, On the discretization of spatially continuous systems with quadratic and cubic non-linearities, JSME Int. J. 41 (1998) 510-531.

[25] G. Rega, W. Lacarbonara, A.H. Nayfeh, C. Chin, Multiple resonances in suspended cables: direct versus reduced-order models, Int. J. Nonlinear Mech. 34 (1999) 901-924.

[26] W. Lacarbonara, A. Paolone, F. Vestroni, Elastodynamics of nonshallow suspended cables: non-linear vibration characteristics, Int. J. Nonlinear Mech. (2006) ISSN: 0020-7462.

[27] W. Lacarbonara, A. Paolone, F. Vestroni, Galloping instabilities of geometrically non-linear nonshallow cables under steady wind flows' 20th ASME Biennial Conference on Mechanical Vibration and Noise, Long Beach, CA, 25-28 September 2005.

[28] A.N. Kounadis, Hamiltonian weakly damped autonomous systems exhibiting periodic attractors, Math. Phys. ZAMP 56 (2006) 324-350.

[29] M. Leipholz, Stability Theory, Academic Press, New York, 1970.

[30] G. Hermann, S. Nemat-Nasser, Instability modes of canitilevered bars induced by fluid flow through attached pipes, Int. J. Solids Struct. 3 (1967) 39-52.

[31] G. Hermann, Stability of equilibrium of elastic systems subjected to non-conservative forces, Appl. Mech. Rev. 20 (2) (1967) 103-108.

[32] A.H. Nayfeh, Introduction to Perturbation Techniques, WileyInterscience, New York, 1991.

[33] Wolfram Research: Mathematica, rel. 5.2, 2005, 〈http://www.wolfram. com $\rangle$.

[34] A.P. Seyranian, A.A. Mailybaev, Multiparameter Stability Theory with Mechanical Applications, World Scientific Publishing, Singapore, 2003. 